\title{
Interactive video over ATM: state of the art
}

\author{
Bing Zheng ${ }^{\mathrm{a}, *}$, Mohammed Atiquzzaman ${ }^{\mathrm{b}, 1}$ \\ ${ }^{\mathrm{a}}$ New Focus Inc., 5215 Hellyer Avenue, San Jose, CA 95138, USA \\ ${ }^{\mathrm{b}}$ School of Computer Science, University of Oklahoma, Norman, OK 73019-6151, USA
}

Received 7 August 2002; accepted 8 August 2002

\begin{abstract}
Multimedia is an emerging service which integrates voice, video and data in the same service. With the progress made in high speed large capacity multimedia servers, high speed networks, QoS, service guarantees and set top boxes, it is currently possible to cost effectively and efficiently carry multimedia over high speed networks. This paper surveys the progress made, and the future of efficiently carrying multimedia over the emerging Asynchronous Transfer Mode networks.
\end{abstract}

(C) 2002 Elsevier Science B.V. All rights reserved.

Keywords: Video on demand; Asynchronous transfer mode; Quality of service

\section{Introduction}

Most of the networks which are currently in use have been developed for data applications. For example, the TCP/IP network only offer best effort service, and hence is not suitable for real time services such video. However, recent efforts, such as Integrated and Differentiated Service at the IETF, are underway to incorporate service guarantees in TCP/IP based networks. As a result, there has been some preliminary work on transporting video over the Integrated Service $[1,2]$. In contrast to legacy data networks, the Asynchronous Transfer Mode (ATM) network has been designed to support both real and non-real time applications making it very suitable for transporting multimedia. It is expected that applications such as video on demand and video conferencing over high speed network will be the most active services over ATM networks. This is evidenced by the strong interest in the standardization effort in carrying multimedia over ATM networks. ATM Forum has already released its standard on transporting compressed video over ATM backbone networks [3], and IEEE Communication Magazine has contributed a feature topic on Video on Demand (VoD) [4-6].

$\mathrm{VoD}$ enables a user to order video from a server. By using set top boxes, a user can perform VCR-like interactive

\footnotetext{
* Corresponding author.

E-mail addresses: zhengbin@ieee.org (B. Zheng), atiq@ou.edu (M. Atiquzzaman).

1 Tel.: +1-405-325-8077; fax: + 1-520-962-8422.
}

functions such as play, fast forward, fast rewind, pause, etc. The following four basic elements are crucial in realizing a VoD service.

- Video Server: a high capacity video server which can be randomly accessed at a high speed as video sources;

- High Speed Network: a high speed network which can serve as a transport channel to support a large number of users.

- Set Top Boxes: a cost effective set top box with reasonable amount of buffering and also has a video decoder, and

- Cost Effective Service: a cost effective service which can offer acceptable quality of service.

The concept of $\mathrm{VoD}$ was presented more than ten years ago [7] where the author discussed the possibility of transporting video over fiber with grade of service (GOS). With the development of broadband telecommunication and network technology, the concept has become a reality. In Refs. [8,9], the authors discussed a broadband network based system architecture for a large scale VoD. Implementation of VoD systems over ATM packet networks have been reported in Refs. [10,11].

The development of the high speed ATM network and Redundant Array of Inexpensive Disk (RAID) which can be used as high speed video servers to server many users have made it possible to run VoD over networks. ATM offers a number of service categories among which the ABR service 
category offers cost effective service to enable VoD-based entertainment, education, and multimedia information services.

The objective of this paper is to study the progress made and the future of running VoD over ATM networks. We have discussed the basics of ATM and interactive video, and the suitability of the various ATM service categories for carrying interactive multimedia. Strategies and results of operating interactive multimedia over ATM networks have been presented.

In Section 2, we discuss ATM networks and its service categories, followed by $\mathrm{VoD}$ and video compression techniques in Sections 3 and 4. The suitability of ATM for video transmission is presented in Section 5 followed by recent studies on carrying highly interactive $\mathrm{VoD}$ over the ABR service of the ATM network in Section 6. Our concluding remarks are given in Section 7.

\section{ATM technology}

ATM is a totally different transfer technology from the traditional Synchronous Transfer Mode (STM) technology which has been employed for decades in telecommunications. ATM is based on statistical multiplexing which allows carrying real-time and non-real time services in the same network. It also results in a great advantage in dealing with bursty data like compressed video. With the coming of the 21 st century, the computer and communication network is becoming more and more important in industry and our daily life. There are four reasons which make ATM advantageous over other networks.

- The difference in the nature of the traffic, currently separate networks are used to carry voice, video and data. This results in a huge burden in the construction, maintenance and managing the different types of networks. With the standardization of Broadband Integrated Service Digital Network (B-ISDN), we can expect to form a universal network which will integrate the three type of networks into one. ATM allows transmission of voice, data and video simultaneously in one network.

- Several technologies, such as Ethernet, Token Ring and FDDI have been developed and deployed in Local and Wide Area Networks. Because of the difference in the network technologies, interconnecting the LANs and WANs to form a large scale nation wide and even international networks is complex and difficult. ATM can be used as LANs and WANs and even as the backbone in LAN/MAN interconnection.

- Since the present networks use different technologies, they use different standards and protocols. For the ease of developing applications, a world wide standard is needed to allow interoperability of information, regardless of the type of information and the type of system. ATM offers a single standard for all types of applications.

- Finally, with the coming of the multimedia era, in the near future we will see video on demand in entertainment, education, shopping, etc. With ATM having several service types to satisfy different application and requirement of service quality, ATM is an ideal solution for multimedia over high speed networks.

\subsection{ATM service categories}

ATM Forum has defined and standardized four types of services: Constant Bit Rate (CBR), Variable Bit Rate (VBR) including real time VBR and non-real time VBR, Available Bit Rate (ABR) and Unspecified Bit Rate (UBR). Their specifications and characteristics are shown in Table 1.

Although the CBR and VBR services offer quality of service (QoS) guarantees and have simple bandwidth management, they are expected to be higher in cost than the ABR service. Because of the high cost, they may not be suitable for entertainment type of applications. In contrast, with ABR service, we can obtain acceptable QoS with very low cost, and high network utilization. Hence, the ABR service offers a cost effective network solution for applications such as VoD. Fig. 1 shows typical trunk utilization for MPEG video transported over the three ATM service categories [12].

\subsection{Quality of service}

One of the strong features of ATM is its quality of service guarantees like delay, cell loss and bandwidth guarantees. Real-time voice and video (and applications such as VoD) require stringent delay and bandwidth guarantees for acceptable picture quality which makes ATM very suitable for carrying multimedia traffic.

\section{Video on demand}

Video on demand (VoD) enables users (clients) to request a video through a network from a video server with a

Table 1

Characteristics of the three service types

\begin{tabular}{lllllll}
\hline Service type & Parameters during connection setup & Bandwidth renegotiation & Bandwidth guarantee & QoS guarantee & Bandwidth utilization & Cost \\
\hline CBR & Constant rate & No & Yes & Yes & Very low & Very high \\
VBR & Range of rate & No & Yes & Yes & Low & High \\
ABR & MCR, PCR, ICR, TBE, RIF, RDF & Yes & Only MCR & Acceptable & Very high & Very low \\
\hline
\end{tabular}




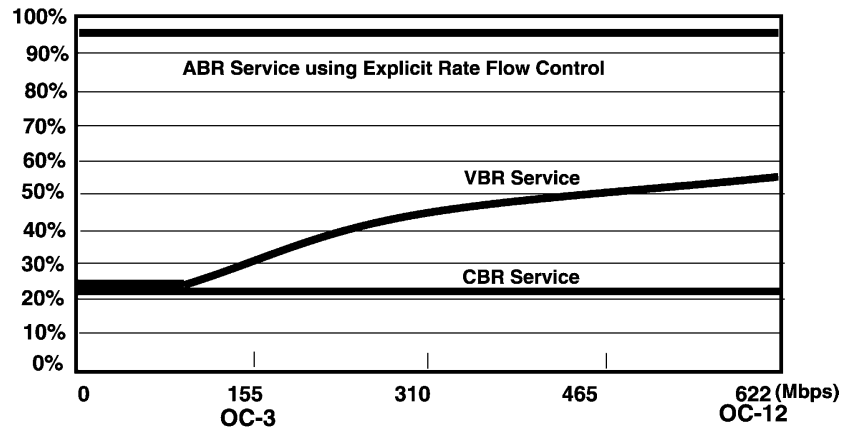

Fig. 1. Bandwidth utilization for transmission of MPEG video over the three service types of ATM.

large scale video data base. Generally, the VoD system consists of a video server and a client connected through a network. A VoD system running over an ATM network is shown in Fig. 2:

Depending of the usage pattern, VoD systems can be classified into three categories:

- Near Video on Demand (NVoD): In NVoD, the client/user sends a request to the video server and waits for the server to broadcast the requested video at a later time. During the playback, the user is a passive viewer [7]. This is somewhat like a cable TV, the difference is that the client/user can request what he/she wants. Therefore, the server/client operation model and principle is simple.

- Interactive Video on Demand (IVoD): Interactive video on demand has VCR-like functions between the server and the client $[8,9,13]$. The client/user sends request to the video server, and the client receives the video from the server and starts the playback. A buffer is used at the client to smooth out fluctuations in the network bandwidth and allow continuous viewing. While in playback, the client/user can perform fastforward (FFW), fastbackward (FBW) or pause operation.

There are two main issues to be solved in IVoD systems. The first is the client/user buffer requirement. Since the normal playback and FFW/FBW have different data consumption rates, the buffer needs to be properly dimensioned to prevent underflow or overflow at the client/user buffer. Because of the the interactive nature of the client, the buffering requirement is decided by the video rate, the network parameters and the level of

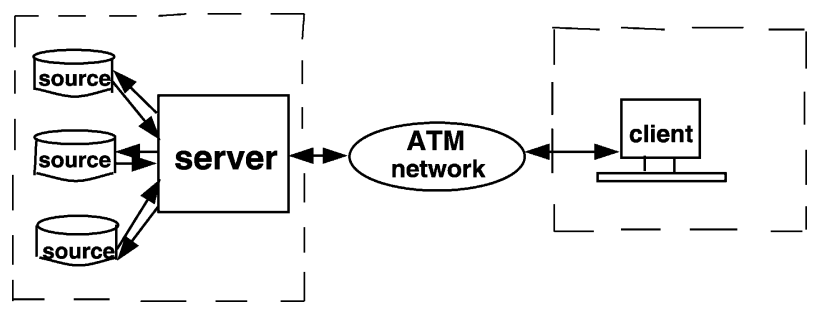

Fig. 2. A video on demand system. interaction. The second issue is the required network bandwidth and network parameters. To meet the QoS of the user, the server needs different network bandwidths at different times.

- Highly Interactive Video on Demand (HIVoD): HIVoD has a much higher level of interactivity between the server and client/user [14]. HIVoD occurs very frequently in educational applications. In $\mathrm{HIVoD}$, the client/user frequently performs FFW/FBW operation which results in two consequences: the first is that the client/user buffer will be depleted/refreshed frequently; to prevent the buffer from starvation, the client/user buffer size must be carefully dimensioned. The second is that the server will frequently ask for different bandwidths in order to minimize the startup delay. It will ask for a high bandwidth in FFW/FBW, while in playback, it needs a relatively low bandwidth.

From the above, we can see that if the bandwidth request of the server cannot be met by the network, the QoS will be affected in the use of both IVoD and HIVoD.

\section{Video compression}

Video is a set of related pictures which are presented to us by a display device. Each picture is formed by a large number of elements called pixels which contain object information such as shape, brightness, color, contrast, etc. The number of pixels in a single picture determines the resolution of the picture. Therefore, to represent a picture with acceptable resolution, we need a large number of bits. In video terminology, each picture is called a frame. Because of the persistence of vision of the human eye, we see a continuous moving picture when a consecutive set of pictures are presented at a certain speed.

The NTSC standard in the USA consists of $480 \times 480$ pixels per frame at a rate of 29.97 frames/second. To transmit the uncompressed video in NTSC standard, we need a channel capacity of $168 \mathrm{Mbps}$ (note that an OC-3 trunk has a channel capacity $155 \mathrm{Mbps}$ ). It is therefore not practical to transmit uncompressed video through a telecommunication network.

Video signals contain a significant amount of redundancy. The first is the statistical redundancy where each frame has information which is related to its adjacent frames and can thus be borrowed from adjacent frames. The second type is the perceptual redundancy which results from signal which cannot be perceived by human eyes. Therefore, it is possible to reduce the bit rate of video by compressing the video with little or no effect on video quality. Currently, there are two widely used compression techniques, MPEG and JPEG, which are described below. The MPEG standard is much more suitable for video compression in a video on demand system, while JPEG is ideal for still image compression. 


\subsection{MPEG digital video compression standard}

Moving Picture Experts Group (MPEG) was established by the International Standard Organization (ISO) to work on internationally recognized world-wide standards for digital video compression. MPEG compression is accomplished in four basic steps: pre-processing to filter out perceptual superfluous information; Temporal Prediction and Motion Compensation takes advantages of the fact that each frame in a video may be similar to the preceding and following frames; finally, the Quantization Coding converts the coefficients of the Discrete Cosine Transfer (DCT) of the residual difference between frames into more compact digital representation.

MPEG frames are divided into three type: an I frame (or intra-frame) acts as a reference for predicting subsequent frames. I frames have the highest data rates. A P frame (or predicting frame) is predicted from the nearest I or P frame; a B frame (or bidirectional frame) uses the information from its adjacent I and/or P frame. MPEG frames are arranged in groups called Group of Pictures (GoP) which is represented by $\mathrm{M} m \mathrm{~N} n$ meaning that there are $n$ frames in a GoP, of which there is one I frame at the beginning of the GoP, followed by $m-1$ number of $\mathrm{B}$ frames between I and $\mathrm{P}$ frames (see Fig. 3). For example, a M3N9 GoP has the structure IBBPBBPBB. The MPEG standard is especially suitable for motion picture. Typically, MPEG technology has a very high compression ratio ranging from 20 to 100 for video. ISO has completed the standardization for MPEG-2 which has features such as random access, trick modes, multicast to many terminal type, multiple video/audio and compatible stereoscopic 3D pictures. The MPEG-2 standard will be widely used commercially in the coming years.

\subsection{JPEG digital video compression standards}

A second widely accepted video compression technology is Joint Photographic Expert Group (JPEG) which has been developed for color still-image compression. Similar to MPEG, JPEG also employs lossy compression scheme to

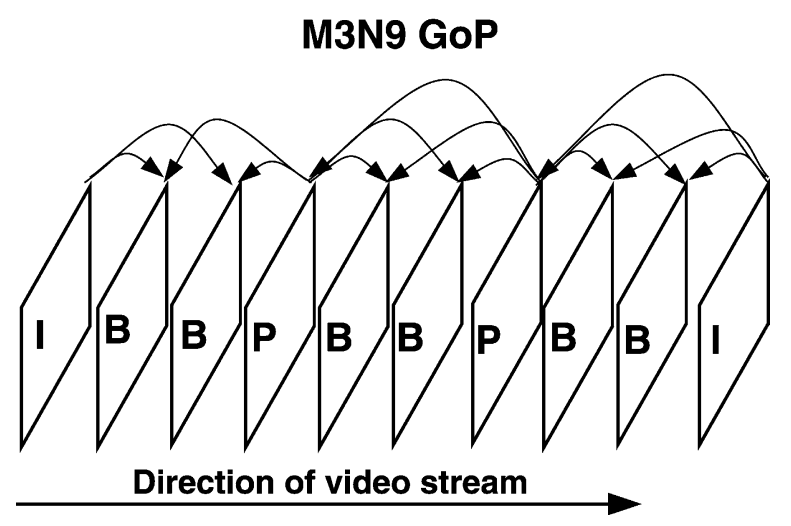

Fig. 3. Group of pictures (GoP) in MPEG-2.

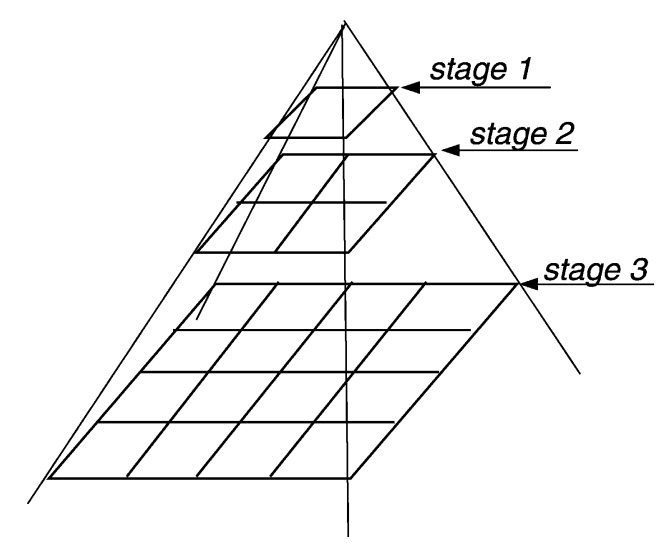

Fig. 4. Hierarchical image representative in JPEG.

filter out superfluous information in images, encode the residual information based on DCT. Unlike MPEG, the JPEG standard has been developed for still-photographic image compression; so, there is no frame group structure. Instead, JPEG employs hierarchical image representation in which a single JPEG still-image is composed by superposition of a number of images of varying resolution as shown in Fig. 4. The resolution is doubled at every stage, and each stage encodes only its difference from the upper one.

\section{ATM for video transmission}

The following sections describe techniques to transport video over the different services of an ATM network.

\subsection{Video over ATM with CBR}

A Constant Bit Rate (CBR) service requires specifying the Peak Cell Rate (PCR) of the connection, and does not allow bandwidth renegotiation during the connection. For video over CBR, the PCR should be equal to the highest bit rate of the video stream if no traffic shaping is used. However, because of the bursty nature of compressed video, the bandwidth utilization for CBR is quite low. The connection cost is therefore, very high which makes it very cost ineffective for popular applications such as home videos and games. The relationship between transmission rate and the client buffer size to avoid underflow or overflow for CBR has been reported in Ref. [15].

The paper by Kang et al. [16] discussed a 2-phase smoothing technique to transmitting the video over a CBR connection. The objective is to shape the traffic to reduce the bandwidth requirement of a CBR connection and thereby achieve higher bandwidth utilization and guaranteed QoS.

\subsection{Video over ATM with VBR}

To reduce the connection cost and improve the bandwidth utilization, it is possible to transport video over 
the VBR service category. A VBR connection is specified by a PCR and a Sustainable Cell Rate (SCR). All bandwidth negotiations have to be done during the connection set up phase. Previous work on video over VBR have dealt with the following issues:

Traffic Shaping and Rate Control: Traffic shaping and rate control aims is to smooth the video stream to reduce the burstiness of video stream. Unfortunately, this results in jitter due to the expansion of the transmission time. Buffers are usually used as compensators by accumulating the expanded (spread) data. In Refs. [17,18], the authors discussed traffic shaping of VBR traffic with a leaky bucket. To reduce the shaping delay and delay variation, the shaper buffer size and the rate control mechanism has to be properly chosen. Super leaky bucket (S-LB) was discussed in Ref. [19], where the traffic is handled based on their QoS requirements. QoS requirements are met by having separate queues for different traffic. The simulation results demonstrate the improvements in the service level such as delay, delay variation and cell loss.

Bandwidth Allocation/Management: To utilize the bandwidth of the network efficiently, Krunz, et al. [20] discussed the sharing of the trunk capacity among a large number of multimedia streams. Bandwidth sharing can increase the bandwidth utilization and decrease the burstiness of the traffic, and statistical multiplexing is most suitable for realtime video transmission [21]. In Refs. [22-24], the authors have investigated the feedback control scheme in bandwidth allocation and management. They have shown that feedback control is effective in transmitting video over an ATM network. For stored video, allocation of bandwidth by predicting future requirement based on current parameters is discussed in Ref. [25]. Since the traffic characteristics of stored video can be anticipated very well, this scheme performs very well.

Congestion Control: Since ATM is based on statistical multiplexing, congestion occurs when the sum of the bandwidths of a number of video sources exceed the total capacity of the network. Congestion degrades the performance of the network and affects the QoS of the connections. Kawahara [26] proposed and analyzed a reactive congestion control algorithm.

Buffer and Memory Requirements: Because of the bursty nature of video traffic, a buffer (to smooth out the fluctuations in the traffic) in an integral part of a video client. It is very important to properly dimension the size of the buffer. If it is too small, there will be overflow/underflow; too large a buffer size increases the cost of the client. Buffer and memory requirements are discussed in Refs. [27, 15]. The authors in Ref. [28] proposed a source model to evaluate the play-out buffer and end to end delay based on discrete Markov chain.

Source Model and Behaviors: A proper video source model is essential for the analysis of VoD systems. Video models fall into three categories: the first uses the Autoregressive Processes [29,30], the second uses Markov chains $[31,32]$ and the third uses a combination of the above two methods [33]. In Refs. [34,35], the authors presented the statistical characteristic of MPEG-2 video source. It is found that MPEG compressed video has correlation relationship between its frame, and the frame size has Gamma distribution.

Coding Technology: In Ref. [36], the authors studied the relationship between the coding method and the traffic characteristics of video. It was found that the traffic characteristics of a video stream is very sensitive to the coding algorithm used.

Quality Control: Quality control mechanism for video over ATM with VBR service has been discussed in Ref. [37]. By establishing the quantitative relationship between picture quality and video source rate to guarantee QoS for different allocated bandwidth, it is found that to maximize the picture quality, the video source rate control should be implemented on both the basic layer stream and enhanced layer stream of the video.

\subsection{Video over ATM with $A B R$}

Unlike CBR and VBR connections, when an ABR connection is set up, the server negotiates Minimum Cell Rate (MCR), PCR and a number of other parameters. During the entire connection, only the MCR is guaranteed by the network. After a connection has been established, a source (video server in our case) can ask for bandwidth between MCR and PCR. If the network has enough bandwidth it will allocate the requested bandwidth to the server; if there is not enough bandwidth available, the network will offer less than the requested amount of bandwidth. In ABR connections, the Allowable Cell Rate (ACR) varies between the PCR and MCR.

Work on transporting video over ABR is at its infancy. In Ref. [38], closed-loop feedback was applied to control congestion, and a non-zero MCR was set to obtain the guaranteed service. In Ref. [34], the authors discussed the explicit rate feedback control scheme to transport compressed video, and investigated the effect of network round trip time on the efficiency of the feedback scheme. The authors in Ref. [39] discussed QoS and multipoint support for video over ATM with ABR service. It was found the ABR service has attractive features in terms of low cell loss and short queuing delay. In Ref. [40], traffic management for multimedia over ATM was discussed, especially video transmission over ABR services. It was concluded that ABR services offers close loop feedback control mechanism for dynamic bandwidth allocation, congestion control, rate control, and the QoS.

\section{Video on demand over ABR}

Until now, the work on $\mathrm{VoD}$ over ABR has been very limited. Because, the ABR service does not guarantee a high 


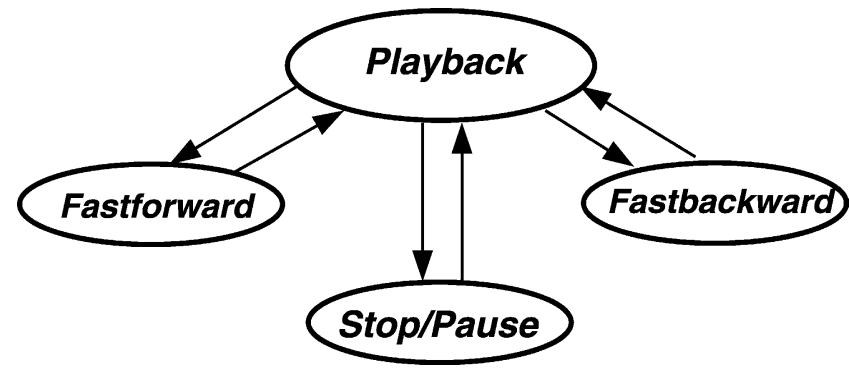

Fig. 5. Illustration of the client operation mode.

bandwidth to a video server, and the quality of service for video is sensitive to delay, buffering requirements and network congestion are two aspects which must be investigated. The first step toward these problems is presented in Refs. [41,14]. In Ref. [41], a GoP bandwidth negotiation scheme was proposed, and the buffering requirements and QoS under different network congestion status were discussed. In Ref. [14], a fast buffer fillup scheme was presented to reduce the network bandwidth requirements and to simplify the bandwidth allocation management. Optimum dimensioning of client buffer and reducing the startup delay corresponding to different server/ client interactivity level were also investigated. Some of the results of preliminary investigations on determining the buffer size and network parameters from the above studies are given below. The scheme in Ref. [41] uses a method where the bandwidth is renegotiated for every GoP. This results in very frequent bandwidth renegotiation. The second method [14], called the Fast Buffer Fillup (FBF) attempts to fill up the client buffer very quickly after a FFW/ FBW operation by asking for more bandwidth from the network. This also eliminates the problem associated with too frequent bandwidth renegotiation which occurs in the first method.

\subsection{Buffer requirement}

Both for IVoD and HIVoD, because of the burstiness of video and the cost of hardware, buffering is an important issue. For interactive VoD with VCR-like functionality, we can illustrate the operation mode of a client by the state diagram in Fig. 5. By using the Real Time Dynamic Equilation (RTDE) and Markov chain analysis [14], we obtain the minimum client buffer size for fast buffer fillup scheme as:

$C_{\text {min }}^{C}=2(k-1) T_{d} E[\lambda]_{f}+T_{f}\left(E\left[\beta_{I}\right]-E[\beta]\right)$

where $E[\lambda]$ is the expected rate of video, $k$ is the speed factor of FFW/FBW, Tf is the time duration for a single frame of MPEG-2 video, $E\left[\beta_{I}\right]$ and $E[\beta]$ are the I-frame rate and the average rate, respectively, of the MPEG-2 compressed video, and $T_{d}$ is the fixed round trip time (FRTT) of the network. When the network bandwidth is negotiated at every GoP, only the first term in Eq. (1) is needed. The numerical results corresponding to different level of interactivity and FRTT are presented in the rest of the section.

For the FBF scheme, we notice that the minimum client buffer size increases linearly with the FRTT because more buffering is required to maintain the display when the round trip time is large (Fig. 6). Note that an M3N9 GoP has a higher bit rate than an M3N15 GoP. For large values of FRTT (corresponding to

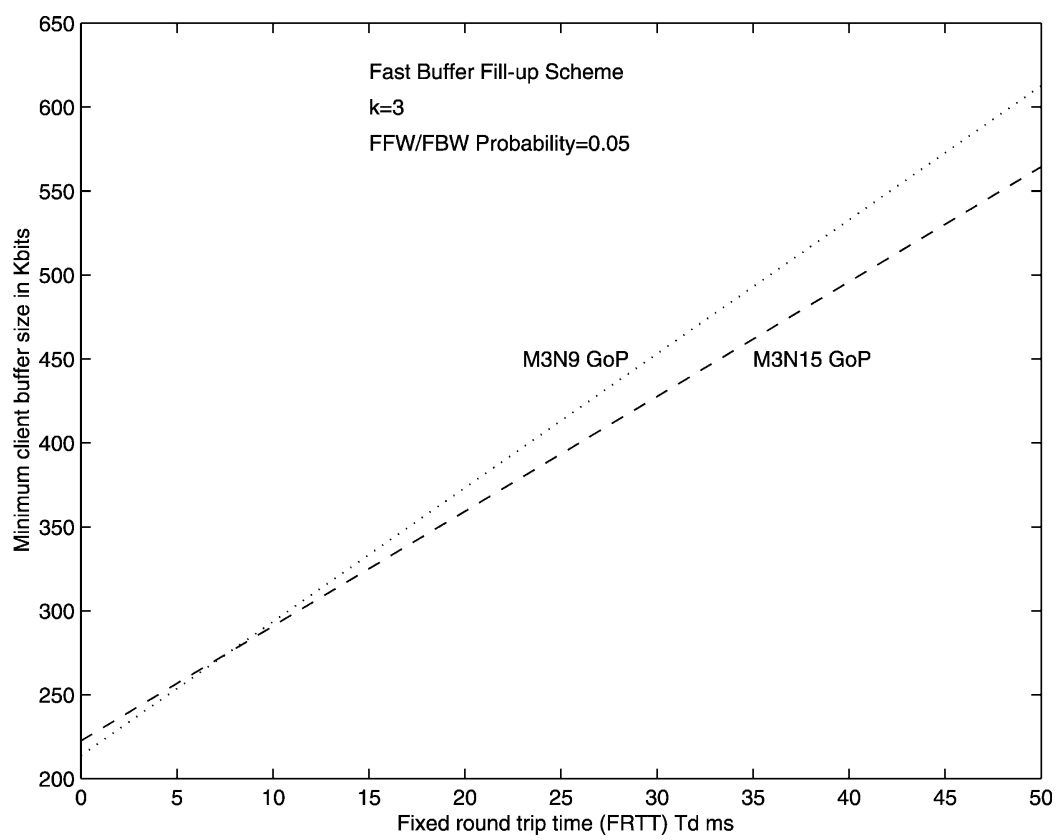

Fig. 6. The minimum client buffer size versus the FRTT for the fast buffer fill-up (FBF) scheme. 


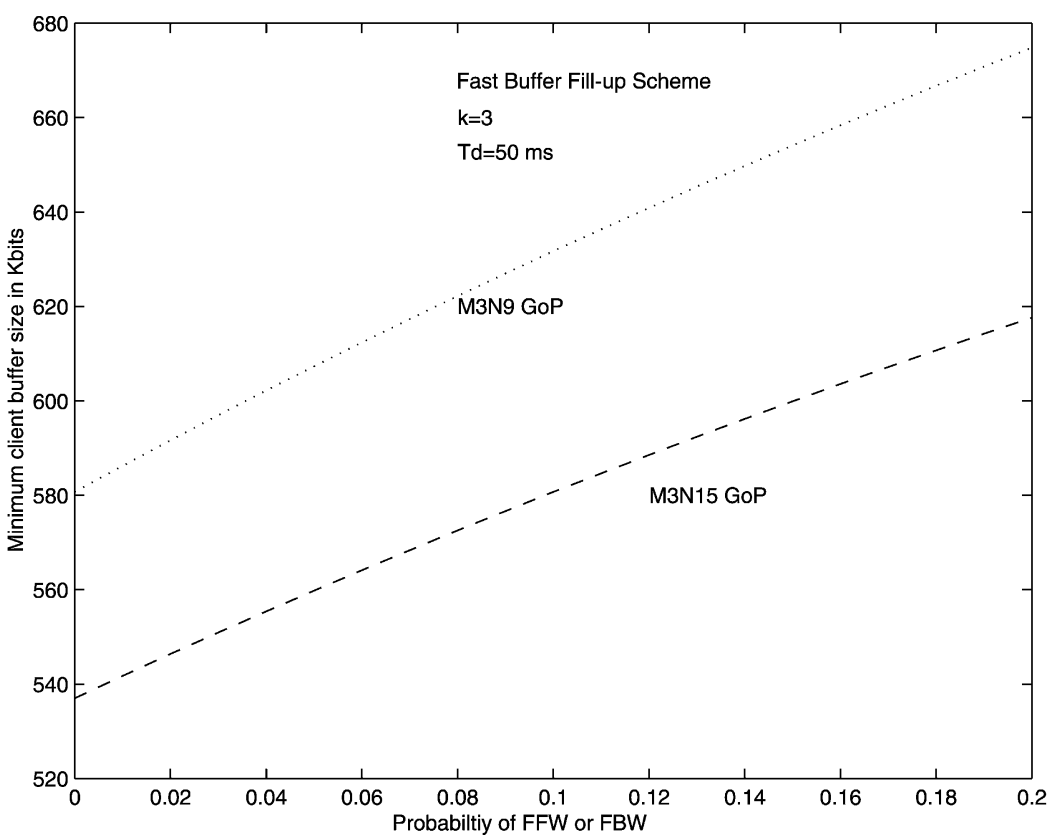

Fig. 7. Minimum buffer size of client versus the FFW or FBW level fort the FBF scheme.

a WAN/MAN), the buffer requirement for M3N9 GoP is larger than for M3N15 GoP. On the contrary, for small FRTT (corresponding to a LAN), M3N15 GoP requires a larger buffer than M3N9. This is explained by the fact that for a small FRTT, the first term of Eq. (1) is small, and the buffer size is dominated by the second term of Eq. (1) which is the rate difference between the I-frame and the average rate.

Fig. 7 illustrates the minimum client buffer size corresponding to FFW/FBW probability in the range of
0-0.2 with fixed FRTT. The client buffer size increases quasi-linearly with the FFW/FBW probability. Since the M3N9 GoP has a higher data rate than an M3N15 GoP, it requires a larger buffer.

Comparing the two bandwidth renegotiation schemes in Figs. 8-11, we notice that they have similar pattern. In the first scheme, when the bandwidth is renegotiated for every GoP, the buffer size requirement is small at the price of increasing the complexity of network bandwidth management.

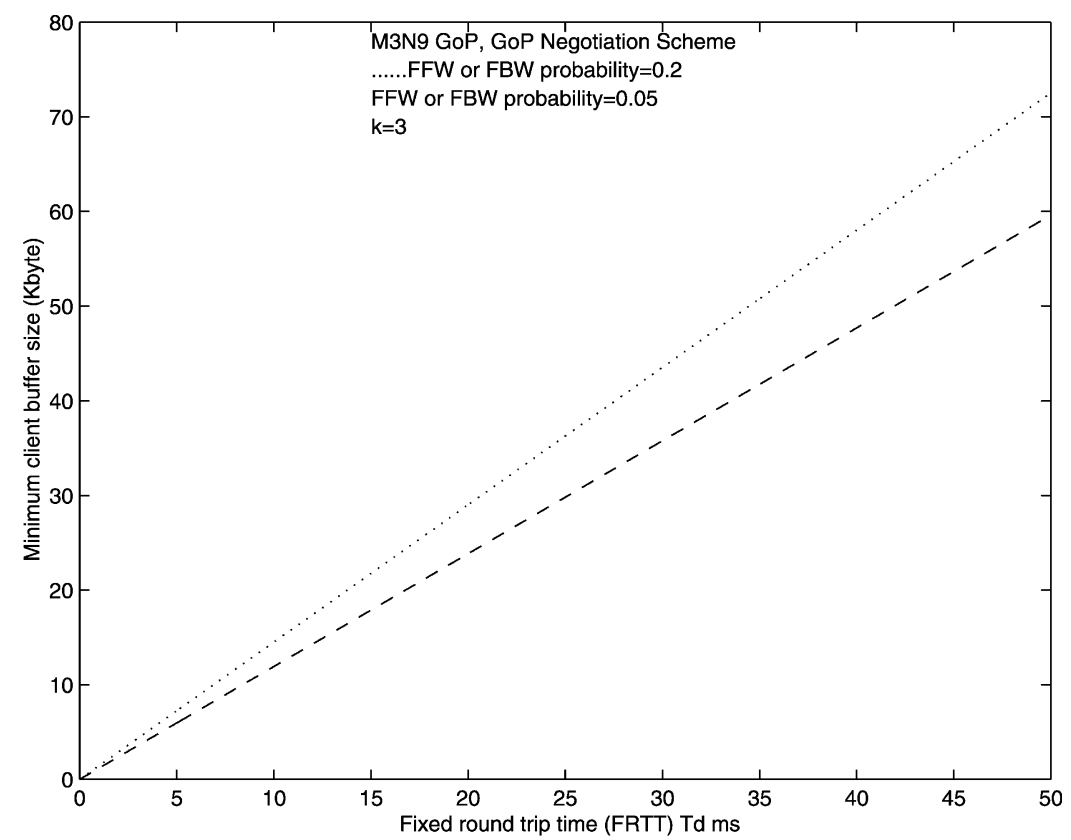

Fig. 8. The minimum client buffer size versus the FRTT for M3N9 GoP with bandwidth renegotiation at every GoP. 


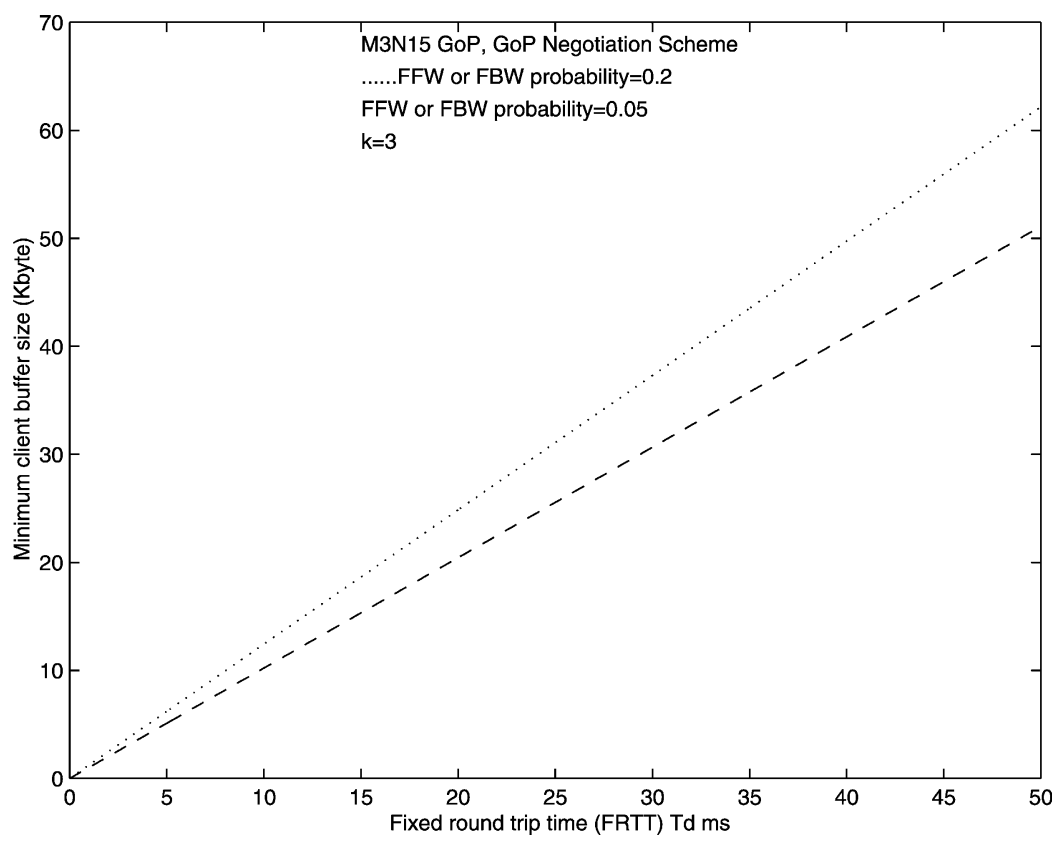

Fig. 9. The minimum client buffer size versus the FRTT for M3N15 GoP with bandwidth renegotiation at every GoP.

\subsection{Network parameter and the QoS}

Transmission of video over ABR requires the determination of MCR and PCR which are related to the particular video being transmitted and has impact on QoS. The average rate of an MPEG-2 video with $\mathrm{M} m \mathrm{~N} n$ GoP can be expressed as:

$E[\beta]=\frac{\beta_{I}+\beta_{P}(n / m-1)+\beta_{B} n=m(m-1)}{n}$

For no overflow/underflow at the client buffer for the entire playback period, the long term dynamic variation of the buffer accumulation should be zero:

$\sum_{\text {all GoP }} \delta($ buffer accumulation of a GoP $)=0$

The expected value of ACR (denoted by $E[\mathrm{ACR}]$ ) must satisfy the following condition:

$$
\begin{aligned}
& \sum_{\text {all GoP }} E[\mathrm{ACR}] n T_{f} \\
& \quad=\sum_{\text {all GoP }} \beta_{I} T_{f}+\beta_{P}(n / m-1) T_{f}+\beta_{B}(m-1) n / m T_{f}
\end{aligned}
$$

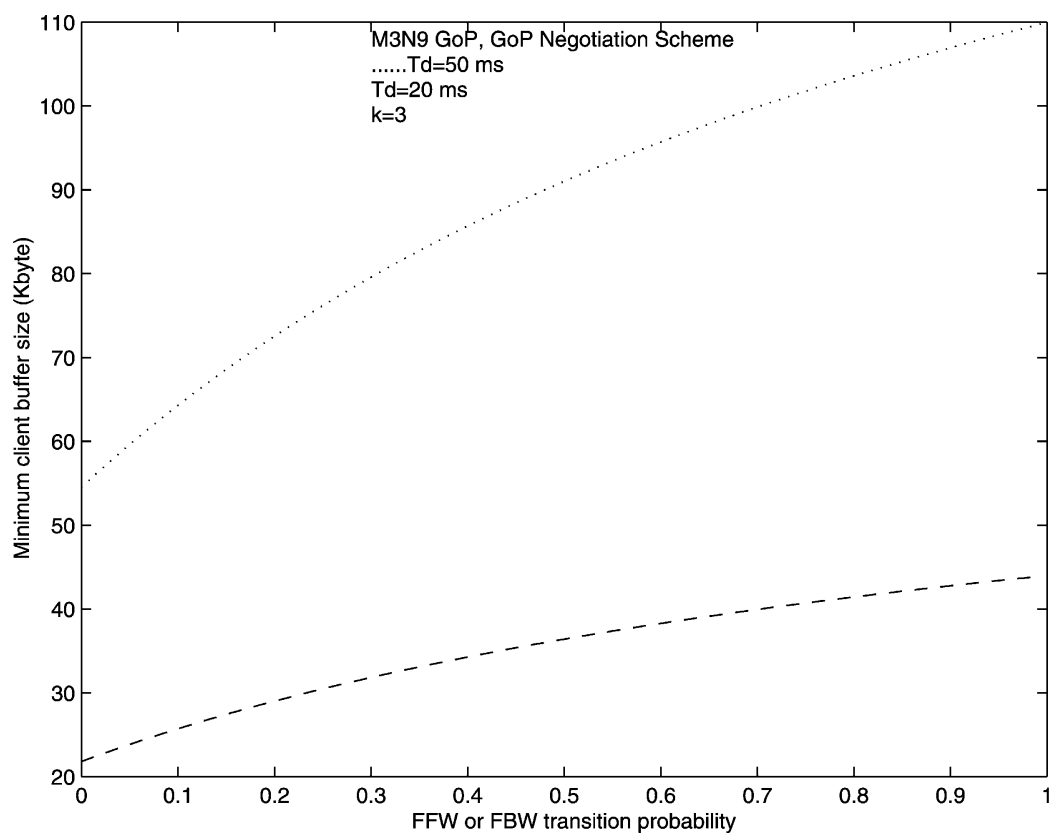

Fig. 10. Minimum buffer size of client versus the FFW or FBW level for M3N9 GoP with bandwidth renegotiation at every GoP. 


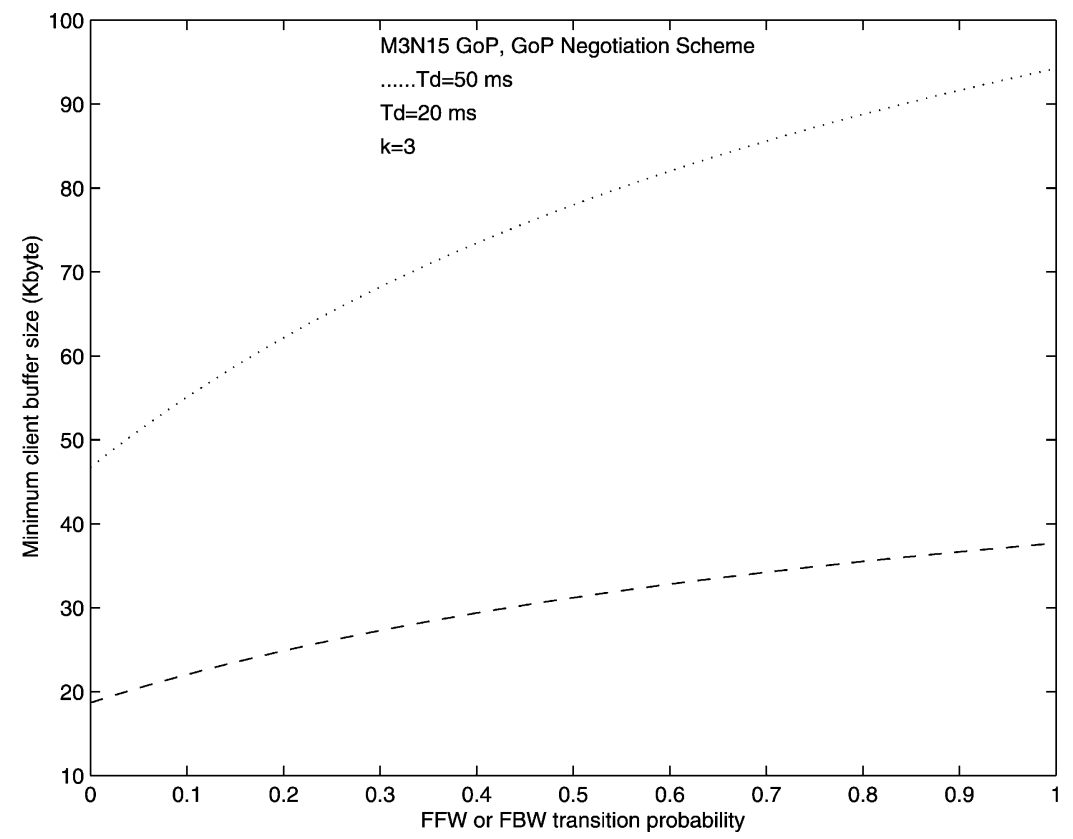

Fig. 11. Minimum buffer size of client versus the FFW or FBW level for M3N15 GoP with bandwidth renegotiation at every GoP.

Therefore, to obtain acceptable QoS, we choose MCR = $E[\mathrm{ACR}]$. In the fast buffer fillup scheme, to fill the client buffer as soon as possible, we set a very high PCR which is several times higher than MCR.

For video on demand, startup delay is an important parameter which affects the QoS. The fast buffer fillup scheme is designed to reduce the startup delay by exploiting the bandwidth renegotiation feature of ABR when the user uses one of the trick modes. We define the startup delay as the time between the user pressing the Playback (from the stop state) to the start of the video play, or the time between the user pressing FFW/FBW to the start of the video. The startup delay $\left(T_{D}\right)$ consists of two parts: a fixed part arising due to the FRTT of the network and the dynamic part $T_{f}$ which is required to fill the client buffer to the minimum level to start playback as shown in Fig. 12.

To evaluate the startup delay, the expected fillup rate during FFW/FBW is employed which can be calculated by the following formula under the assumption that the network has an exponential distribution for bandwidth request $\mu_{r}$ between the MCR and PCR [14].

$E\left[\mu_{r}\right]=\frac{\int_{\mu_{m}}^{\mu_{p}} \mu_{r} P\left(\mu_{r}\right) \mathrm{d} \mu_{r}}{\int_{\mu_{m}}^{\mu_{p}} P\left(\mu_{r}\right) \mathrm{d} \mu_{r}}$

By introducing the parameter $q=\mu_{p} / \mu_{m}$ as the ratio of the PCR to MCR, we obtain the expected allocated rate:

$E\left[\mu_{r}\right]=\left(1 / \alpha+\frac{1-q \mathrm{e}^{-\alpha}}{(q-1)\left(1-\mathrm{e}^{-\alpha}\right)}\right)(q-1) \mu_{m}$
The expected dynamical start delay $E\left[T_{f}\right]$ can be obtained by:

$E\left[T_{f}\right]=\int_{\mu_{m}}^{\mu_{p}} \frac{C_{\min }^{C}}{\mu_{r}} P\left(\mu_{r}\right) \mathrm{d} \mu_{r}$

Since there is no closed form results for the above integration, it can only be calculated by numerical integration. Numerical results for expected fillup rate and expected dynamic start delay are shown in Figs. 13-15.

Fig. 13 shows that the expected ACR during the FFW/ FBW increases linearly with increasing PCR for a constant network congestion. This implies that we can reduce the startup delay by setting a high PCR at connection set up. Fig. 14 shows that the expected ACR during the FFW/FBW decreases rapidly when the network becomes congested resulting in a degradation in the QoS.

The expected value of the dynamic startup delay versus the difference, PCR-MCR, is calculated from Eq. (7) and is shown in Fig. 15. We notice that M3N9 and M3N15 GoPs

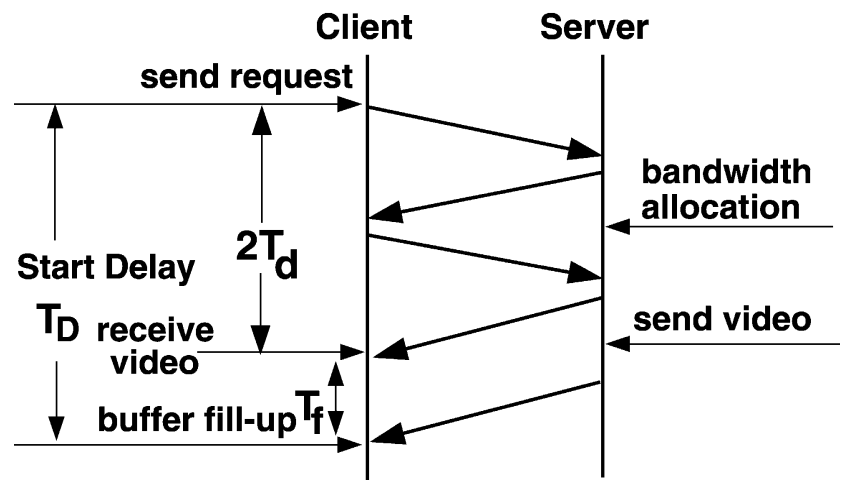

Fig. 12. Illustration of the startup delay. 


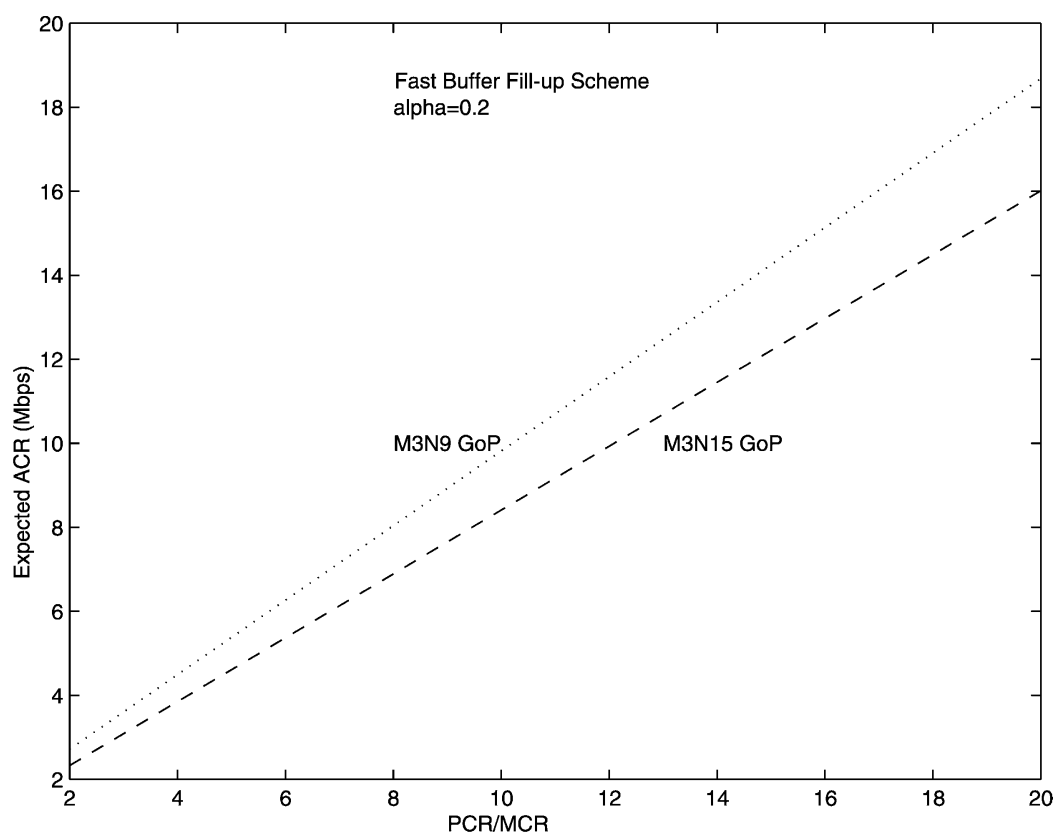

Fig. 13. Expected bandwidth allocation versus PCR/MCR for a constant network congestion status.

have the same dynamic startup delay for the same level of network congestion. Moreover, the dynamic startup delay decreases exponentially with higher values of PCR.

\section{Conclusion}

In this paper we have discussed the progress that have been made so far in the area of multimedia over ATM, taking video on demand over ATM as an example. We have provided an overview of the possible services and their suitability in running video on demand (video in general) over an ATM network. Because of the cost effectiveness of the ABR service of the ATM network, different strategies to run video on demand over ATM has been discussed in detail. Issues like client buffer dimensioning as a function of user interactivity level and effect of round trip time on running video over $\mathrm{ABR}$, traffic and connection parameters

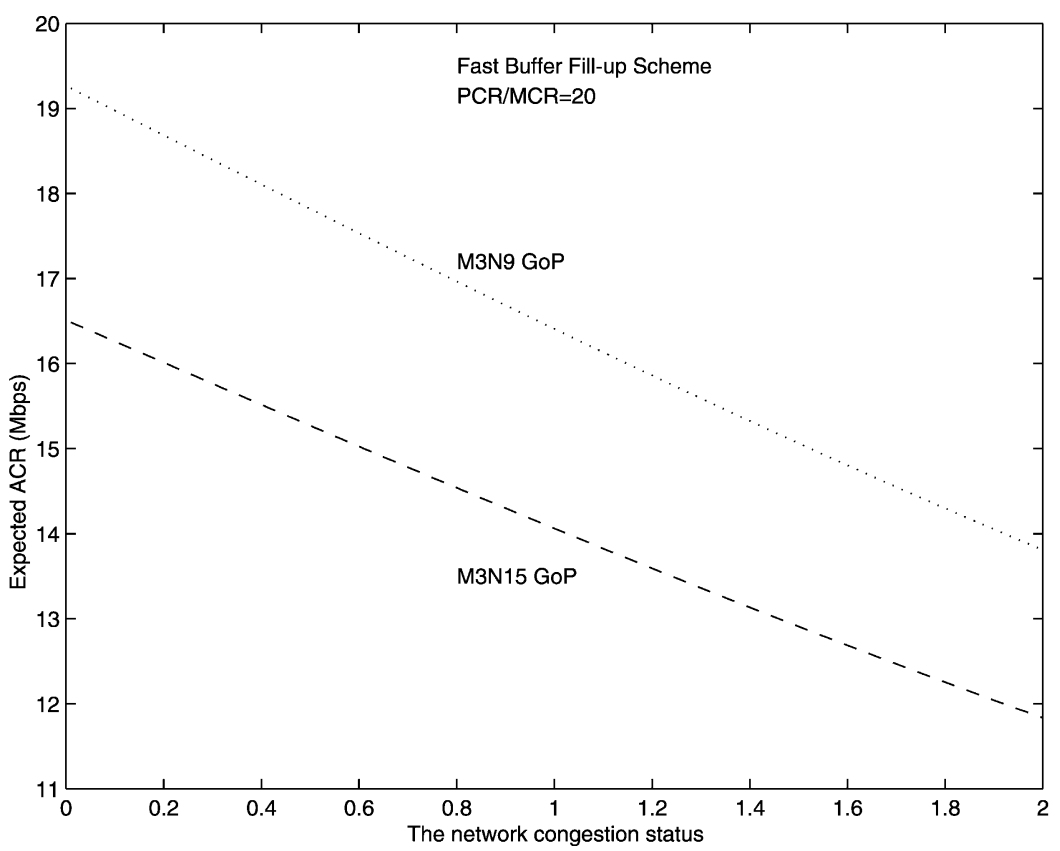

Fig. 14. Expected bandwidth allocation versus the network congestion status for fixed PCR/MCR. 


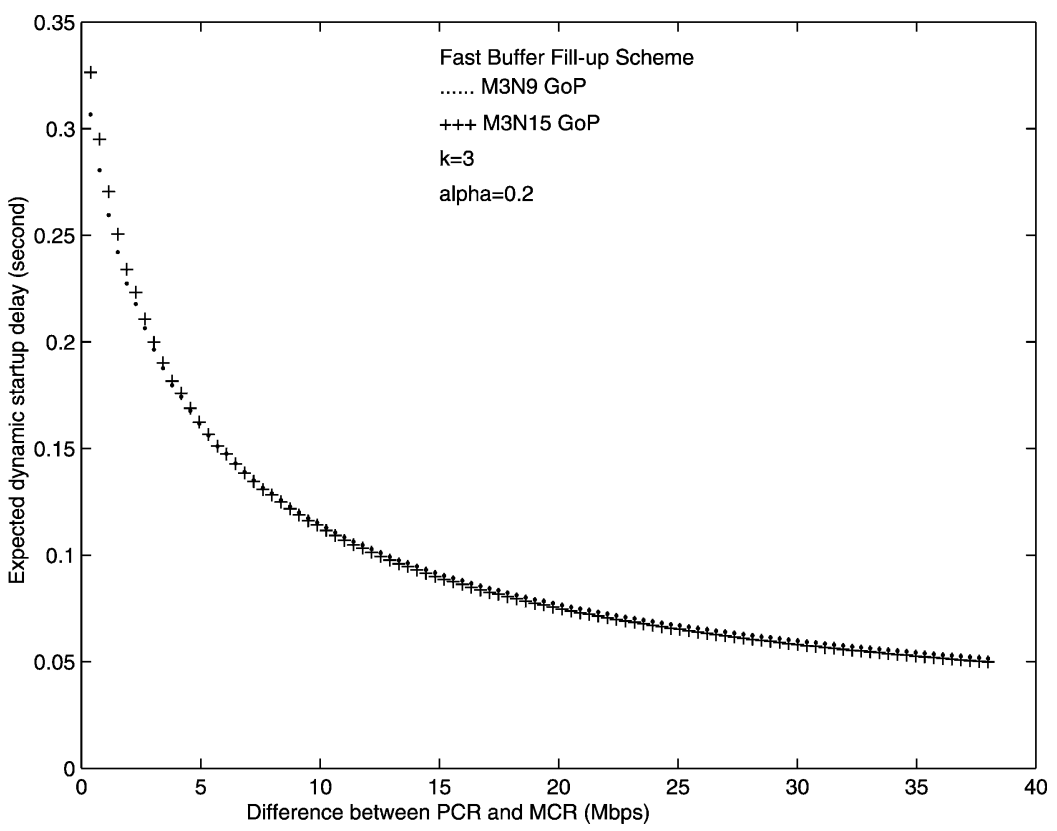

Fig. 15. Expected dynamic startup delay versus the PCR rate.

required during connection setup have been discussed in the paper. We have shown results relating the buffer size required at the client versus the round trip delay in an ATM network. We discussed and compared two operating schemes for running interactive VoD over ATM. In one of the schemes, called the fast buffer fillup (FBF) scheme, complexity of bandwidth allocation and network management is greatly reduced at the price of increasing the buffer size in the client.

The results presented in this paper prove that interactive video can be cost effectively run over the ABR service of ATM. With the standardization of video compression technology, the deployment of high speed ATM network, and the progress in high speed large capacity video server, we have possessed the basic elements to cost effectively offer interactive multimedia services. Further study is required in the area of operating schemes for the client/server model employed in interactive multimedia systems.

\section{References}

[1] M.F. Alam, M. Atiquzzaman, M.A. Karim, Efficient MPEG video traffic shaping for the next generation Internet, IEEE GLOBECOM'99, Rio de Janeiro, Brazil December (1999).

[2] M.F. Alam, M. Atiquzzaman, M.A. Karim, Effects of source traffic shaping on MPEG video transmission over next generation IP network, Eighth IC3N, Boston, USA October (1999) 514-519.

[3] ATM Forum Technical Committee, Video on demand specification 1.0, Technical Report, ATM Forum, December 1995.

[4] D. Deloddere, W. Verbiest, H. Verhille, Interactive video on demand, IEEE Communication Magazine 32 (5) (1994) 82-88.

[5] Y.-H. Chang, D. Coggins, D. Pitt, An open-system approach to video on demand, IEEE Communication Magazine 32 (5) (1994) 68-80.
[6] R.J. Jones, Baseband and passband transport system for interactive video on demand, IEEE Communication Magazine 32 (5) (1994) 90-101.

[7] S. Lederman, Video on demand: a traffic model and GOS technique, GLOBE-COM'86 (1986) 676-683.

[8] W.D. Sincoskie, Video on demand: is it feasible?, GLOBECOM'90, San Diego, CA (1990) 201-205.

[9] W.D. Sincoskie, System architecture for a large scale video on demand, Computer Networks and ISDN System 22 (2) (1991) 155-162.

[10] M. Reha Civanlar, G.L. Cash, A practical system for MPEG-2 based video on demand over ATM packet networks and the WWW, Signal Processing: Image Communication 8 (1996) 221-227.

[11] A.D. Jong, K. Hsing, D. Su, A vod application implemented in java, Multimedia Tools and Applications 5 (2) (1997) 161-170.

[12] L.G. Roberts, Can ABR service replace VBR service in ATM network, COM-PCON'95 Conference, Piscatway, New Jersey (1995) 346-348.

[13] F.F.N. Pereira, J.M.S. Nogueira, Transmission of MPEG video over ATM-based networks utilizing dynamic bandwidth negotiation, IEEE MMNS'98, Versailles, France November (1998).

[14] B. Zheng, M. Atiquzzaman, Multimedia over high speed networks: reducing network requirement with fast buffer fillup, IEEE GLOBECOM'98, Sydney November (1998).

[15] J.M. McManus, K.W. Ross, Video on demand over ATM: constant rate transmission and transport, IEEE Journal on Selected Areas in Communications 14 (6) (1996) 1087-1098.

[16] S. Kang, H.Y. Yeom, Transmission of video streams with constant bandwidth allocation, Computer Communications 22 (1999) $173-180$.

[17] M. Graf, VBR video over ATM: reducing network resource requirement through endsystem traffic shaping, IEEE INFOCOM'97, Kobe, Japan April (1997) 48-57.

[18] M. Hamdi, J.W. Roberts, P. Rolin, Rate control for VBR video coders in broad-band networks, IEEE Journal on Selected Areas in Communications 15 (6) (1997) 1040-1051.

[19] D. Gan, S. McKenzie, Traffic policing in ATM networks with multimedia traffic: the super leaky bucket, Computer Communication 22 (1999) 439-450.

[20] M. Krunz, S.K. Tripath, Exploiting the temporal structure of MPEG-2 video for the reduction of bandwidth requirement, IEEE INFOCOM'97, Kobe, Japan April (1997) 143-150. 
[21] M. Krunz, Bandwidth allocation strategies for transporting variable bit rate video traffic, IEEE Communication Magazine 37 (1) (1999) 40-46.

[22] H. Kanakia, P.P. Mishra, A.R. Reibman, An adaptive congestion control scheme for real time packet video transport, IEEE/ACM Transaction on Networking 3 (6) (1995) 671-682.

[23] H. Kanakia, P.P. Mishra, A. Reibman, An adaptive congestion control scheme for real-time packet video transport, ACM SIGCOMM'93 (1993) 20-31.

[24] C.J. Beckman, Dynamic bandwidth allocation for interactive video application over corporate network, IEEE COMPCON'96 (1996) 219-225.

[25] P.P. Mishra, Fair bandwidth sharing for feedback controlled VBR video traffic, IEEE GLOBECOM'95, Singapore November (1995) 1102-1108.

[26] K. Karahara, Y.O.M. Murata, H. Miyahara, Performance analysis of reactive congestion control for ATM network, IEEE Journal on Selected Areas in Communication 13 (4) (1995) 651-661.

[27] A.D. Gelman, S. Halfin, W. Willinger, On buffer requirement for store-and-forward video on demand, IEEE GLOBECOM'91, Arizona (1991) 976-980.

[28] N. Blefari-Melazzi, V. Eramo, M. Listanti, Dimensioning of play-out buffers for real time services in a b-isdn, Computer Communications 21 (11) (1998) 980-995.

[29] R. Grunenfelder, J.P. Cosmas, Characterisation of video codes as autogressive moving average processes and related queueing system performance, IEEE Journal on Selected Areas in Communication 9 (1991) 284-293.

[30] F. Yegenoglu, B. Jabbari, Y.Q. Zhang, Modeling of motion classified VBR video codes, IEEE INFOCOM'92 (1992) 105-109.

[31] D.P. Heyman, A. Tabatabai, T.V. Lakshman, Statistical analysis and simulation study of video teleconference traffic in ATM network,
IEEE Transactions on Circuits and Systems for Video Technolgy 2 (1) (1992) 49-59

[32] P. Skelly, S. Dixit, M. Schwartz, A histogram-based model for video traffic behavior in an ATM network node with an application to congestion control, IEEE INFOCOM'92 (1992) 95-104.

[33] G. Ramamuthy, B. Sengupta, Modeling and analysis of a variable bit video multiplexer, IEEE INFOCOM'92 (1992) 817-827.

[34] T.V. Lakshman, P.P. Mishra, K.K. Ramakrishram, Transporting compressed video over ATM networks with explicit rate feedback control, IEEE INFOCOM'97, Kobe, Japan April (1997) 38-47.

[35] J. Ni, T. Yang, D.H.K. Tsang, Source modelling, queueing analysis and bandwidth allocation for VBR MPEG-2 video in ATM network, IEE Proceedings on Communication 143 (4) (1996) 120-127.

[36] P. Pancha, M. El Zarki, MPEG coding for variable bit rate video transmission, IEEE Communication Magazine 32 (5) (1994) 54-66.

[37] W. Luo, M. El Zarki, Quality control for VBR video over ATM networks, IEEE Journal on Selected areas in Communications 15 (6) (1997) 1029-1039.

[38] B. Ahn, K.-H. Cho, H. Song, J. Park, Design of ratebased congestion control scheme for MPEG video transmission in ATM network, IEEE GLOBECOM'97, Phoenix November (1997) 1690-1694.

[39] B. Vandalore, S. Fahmy, R. Jain, R. Goyal, M. Goyal, Quality of service and multipoint support for multimedia applications over atm abr service, IEEE Communications Magazine January (1999).

[40] B. Zheng, M. Atiquzzaman, Traffic management of multimedia over ATM networks, IEEE Communications Magazine 37 (1) (1999) 33-38.

[41] B. Zheng, M. Atiquzzaman, Video on demand over ATM: system design and networking requirements, ENCOM'98-The Enterprise Networking and Computing'98, Atlanta June (1998) 114-121. 